\title{
Evaluation of the Local Burning Velocity Using DNS Data of Turbulent Premixed Flames
}

\author{
Kazuya Tsuboi, Ryoji Matsugi, Eiji Tomita \\ Graduate School of Natural Science and Technology, Okayama University, Okayama, Japan \\ Email: tsuboi@mech.okayama-u.ac.jp
}

Received 22 June 2014; revised 25 July 2014; accepted 5 August 2014

Copyright (C) 2014 by authors and Scientific Research Publishing Inc.

This work is licensed under the Creative Commons Attribution International License (CC BY). http://creativecommons.org/licenses/by/4.0/

\begin{abstract}
The local burning velocity, which is based on the consumption rate of the unburned mixture, is one of the dominant parameters in turbulent premixed flames. In this study, the evaluating method of the local burning velocity was investigated using DNS data of turbulent premixed flames with different Lewis numbers. The local burning velocity was evaluated by integrating the chemical reaction rates along normal to the flame surface within three kinds of integration ranges that were defined as follows: the range which is defined by the half length of normal to the flame surface between its certain point and the other point crossing the flame surface (Range 1); the range which is defined by the reaction progress variable that the chemical reaction rate along normal to a planer flame surface takes a half of the maximum value (Range 2); the range which is defined by the length of normal to the flame surface between its certain point and the point which has the extreme value of the reaction progress variable (Range 3). As a result, Range 1 and Range 2 were affected by the flame shapes greatly, since the quantities of the integration ranges fluctuated widely dependent on the variations of turbulent premixed flames. Under the conditions of the turbulent combustion in this study, Range 3, which is hardly affected by a flame shape, is considered to be appropriate to the evaluation of the local burning velocity.
\end{abstract}

\section{Keywords}

Local Burning Velocity, Integration Range, Reaction Progress Variable, Turbulent Premixed Flame, Direct Numerical Simulation

\section{Introduction}

The local burning velocity is one of the dominant parameters on the local structure of turbulent premixed flames. 
The local burning velocity is the instantaneous quantity based on the local consumption rate of the unburned mixture [1]. Burning velocity should be the quantity based on the chemical reaction rate because combustion is a kind of chemical reactions. Therefore, the local burning velocity is considered to be the most appropriate burning velocity in terms of the definition, and has been used for many kinds of studies on turbulent premixed flames.

Haworth and Poinsot [2] investigated the effects of the Lewis number, Le, which is defined as the ratio of the thermal diffusion to the mass diffusion, on the local and global flame structure using two-dimensional DNSs of turbulent premixed flames with single-step overall chemical kinetics. They reported that the local burning velocity was affected by the Lewis number, and was more strongly correlated with the flame curvature than with the tangential strain rate. Rutland and Trouvé [3] implemented three-dimensional DNSs of turbulent premixed flames with single-step overall chemical kinetics, and found that the local burning velocity had a strong and nearly linear correlation with the flame curvature for $L e \neq 1$, and had no correlation for $\operatorname{Le}=1$. Chen and $\operatorname{Im}[4]$ investigated correlations of the local burning velocity with the flame stretch rate, flame curvature, and tangential strain rate using two-dimensional DNSs for hydrogen/air turbulent premixed flames with detailed chemical kinetics for different equivalence ratios. Bell et al. [5] explored the correlation of the local burning velocity with flame curvature using two-dimensional numerical simulations based on a low Mach number formulation for different fuels, including hydrogen, methane, and propane. They reported that the correlation varied with the different fuels. Day et al. [6] investigated the detailed behaviour of premixed flames and reported a correlation of the local burning velocity with the flame curvature using the same numerical method for hydrogen/methane/ air premixed flames. Han and Huh [7] carried out three-dimensional DNS investigations of turbulent premixed flames with single-step overall chemical kinetics and examined the temporal variations of the local burning velocity for the different turbulence intensities at different Lewis numbers.

In this study, the effects of the integration range of the chemical reaction rates on the evaluation of the local burning velocity were investigated using DNS data of turbulent premixed flames with different Lewis numbers.

\section{Numerical Method}

\subsection{DNS Data}

The DNS database used for the numerical analysis of the local burning velocity evaluation was constructed with different Lewis numbers. These were Le $=0.8$, termed case Ml; Le $=1.0$, termed case Mm; and Le $=1.2$, termed case Mh. The simulations were carried out using the PRIMEPOWER HPC2500 installed at Nagoya University [8]. Details of the database are given in Table 1, and the computational domain is shown in Figure 1. The governing equations for constructing the database were the conservation of mass, chemical species, energy, and momentum (compressible Navier-Stokes equations), and the equation of state for an ideal gas. The database was constructed using a sixth-order central finite difference scheme in the mean flow direction and a spectral collocation method in the directions perpendicular to the mean flow for spatial discretisation. A third-order three-step

\begin{tabular}{cccc} 
Table 1. The DNS database. & & & \\
\hline Case & Ml & Mm & Mh \\
\hline$\rho_{u} / \rho_{b}$ & 5.00 & 5.00 & 5.00 \\
Le & 0.8 & 1.0 & 1.2 \\
$u_{L}^{0}(\mathrm{~m} / \mathrm{s})$ & 0.523 & 0.523 & 0.523 \\
$\delta_{f}^{0}(\mathrm{~mm})$ & 0.191 & 0.191 & 0.191 \\
$\bar{u}_{\text {in }}(\mathrm{m} / \mathrm{s})$ & 1.406 & 0.992 & 0.840 \\
$u^{\prime} / u_{L}^{0}$ & 1.01 & 1.01 & 1.01 \\
$\lambda / \delta_{f}^{0}$ & 10.7 & 10.7 & 10.7 \\
$l_{t} / \delta_{f}^{0}$ & 18.0 & 18.0 & 18.0 \\
$\operatorname{Re}_{\lambda}$ & 56.7 & 56.7 & 56.7 \\
$\operatorname{Re}_{l t}$ & 95.5 & 95.5 & 95.5 \\
\hline
\end{tabular}




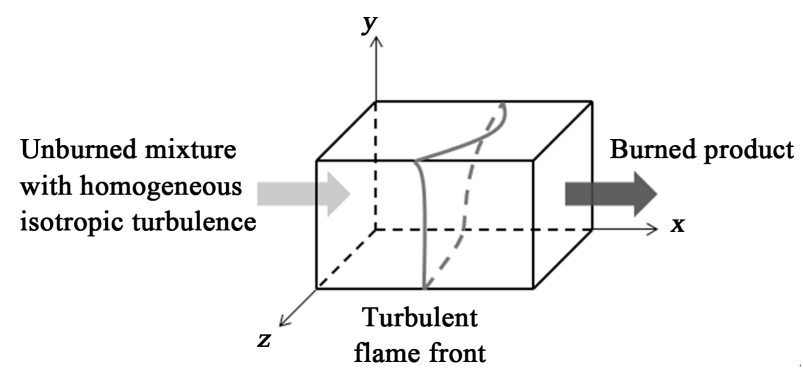

Figure 1. Computational domain.

Runge-Kutta method was used for the time evolution and an overall single-step irreversible reaction was used to describe the chemical kinetics. The inflow and outflow boundaries were described on the basis of Navier-Stokes characteristic boundary conditions (NSCBC) [9] [10], and the lateral boundaries were periodic. The computational domain was $8 \mathrm{~mm}$ in the mean flow direction and $4 \mathrm{~mm}$ in the directions perpendicular to the mean flow; 512 and 128 grid points were used in the respective directions. At the inflow boundary, preliminary calculated homogeneous isotropic turbulence with a cycle of several milliseconds was used, with a mean inflow velocity assuming Taylor's hypothesis of frozen turbulence with a phase shift. Initially, a laminar premixed flame was formed, which grew to form a turbulent premixed flame. The inflow velocity of the unburned mixture was adjusted while monitoring the turbulent burning velocity until the turbulent premixed flame became fully developed and stabilised. The instantaneous turbulent burning velocity varied temporally; however, the time-averaged turbulent burning velocity, which can be measured experimentally, was steady. The database was constructed without changing the inflow velocity. Each case in the database consisted of almost 200 sampled data points at $51.68-\mu$ intervals (which was longer than the DNS time step). The conditions described in the database correspond to the boundary between wrinkled flamelets and corrugated flamelets in the turbulent combustion regime diagram [11]. Further details of the calculation method to construct the DNS database can be found in Nishiki et al. [12] [13] and Nishiki [14].

\subsection{Evaluation of Local Burning Velocity}

The local burning velocity, $u_{L}$ was defined by integrating the chemical reaction rates along normal to the flame surface as follows:

$$
u_{L}=-\frac{1}{\rho_{u} Y_{u}} \int \dot{\omega} d n
$$

where $\rho_{u}$ is the density of the unburned mixture, $Y_{u}$ is the mole fraction of the unburned mixture, $\dot{\omega}$ is the chemical reaction rate, and $n$ is normal to the flame surface. The flame surface was defined by the prescribed isosurface of the reaction progress variable

$$
c_{T}=\frac{T-T_{u}}{T_{a}-T_{u}}
$$

where $T$ is the temperature, $T_{u}$ is the temperature of the unburned mixture, and $T_{a}$ is the adiabatic flame temperature. In this study, the reaction progress variable is prescribed as $c_{T}=0.9$, where the chemical reaction rate has a maximum value along normal to the flame surface.

It is necessary to define the integration range of the chemical reaction rates appropriately when the local burning velocity is evaluated. In the evaluation of the local burning velocity at a given point at the flame surface, the normal to the flame surface at the point may cross the flame surface at another point. To avoid this problem, in this study, three kinds of the integration range were considered. First, the schematic of the integration range, termed Range 1 is shown in Figure 2. At a given point on the flame surface, we evaluated the distance of the normal between the given point on the flame surface and the other point crossing the flame surface, and then obtained half of the distance between these points. This procedure was implemented for all the points where the flame surface intersected with the grid lines, and then the minimum distance was obtained. The integration of Equation (1) was carried out within the obtained distance as one side of the integration range [8]. Second, the schematic of the integration range, termed Range 2 is shown in Figure 3. The conditions of this study lies in the 


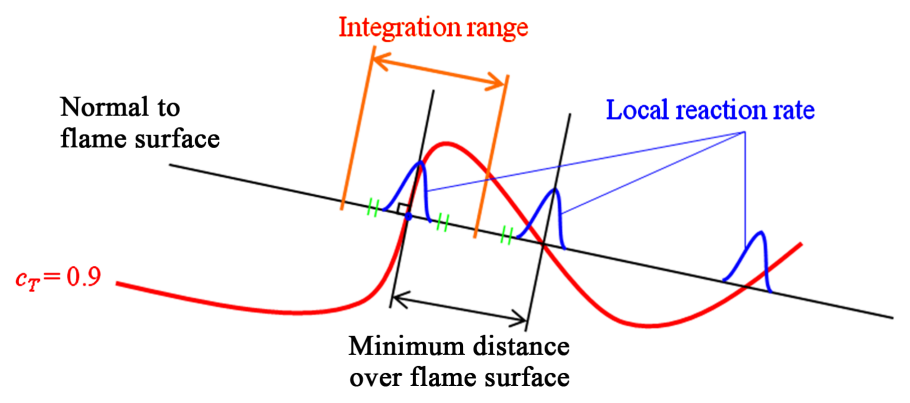

Figure 2. Schematic of the integration range, Range 1.

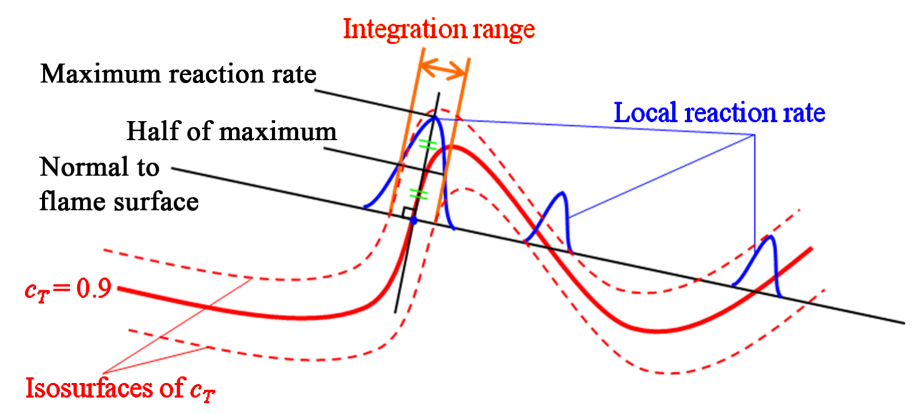

Figure 3. Schematic of the integration range, Range 2.

boundary between wrinkled flamelets and corrugated flamelets in the turbulent combustion regime diagram [11]. In the regime, the turbulent flame is considered to be locally the assembly of laminar flamelets. At a given point on the flame surface, we found the points which has a half of the maximum chemical reaction rate along normal to the flame surface, and then obtained the corresponding $c_{T}$ to the points in the both sides of the unburned mixture and the burned product. Subsequently, the distance between the given point on the flame surface and the $c_{T}$ in the each side was evaluated. This procedure was implemented for all the points where the flame surface intersected with the grid lines, and the minimum distance the each side was set up as the integration range for all the points on the flame surface. Finally, the schematic of the integration range, termed Range 3 is shown in Figure 4. At a given point on the flame surface, we evaluated the distance between the given point on the flame surface and the point which has the extreme value of $c_{T}$ along normal to the flame surface. This procedure was implemented for all the points where the flame surface intersected with the grid lines, and then the minimum distance was obtained. The obtained distance was set up as the integration range for all the points on the flame surface.

All kinds of the integration range were at least the thickness of an unstretched flame, $\delta_{f}^{0}$, and evaluated at each sampled data.

\section{Results and Discussion}

Temporal variations of the integration ranges of the local chemical reaction rates for the local burning velocity evaluation with the different Lewis numbers are shown in Figure 5. In all Lewis number cases, the integration range fluctuated temporally because turbulent premixed flames varied temporally. The temporal variations of the integral ranges increased with Lewis number regardless of the definition of the integration range. When Lewis number is smaller than unity, a flame is unstable and is wrinkled largely. One element on the wrinkled flame surface approaches other element frequently, thus the integration range is limited. On the other hand, when Lewis number is greater than unity, a flame is stable and is wrinkled gently. In this case, the flame elements rarely approach each other. Therefore, a stable flame with higher Lewis number often leads to the wide spread of the integration range. In the case of Range 1, the integration range was wider and fluctuated greatly. This is due to the definition of Range 1 depending on the shape of the flame surface. The integration range in Range 2 was also wider, however, the fluctuation was more gentle than that in Range 1 . In Range 2, the integration range is defined by the corresponding range of $c_{T}$ to the full width at half height of the maximum chemical 


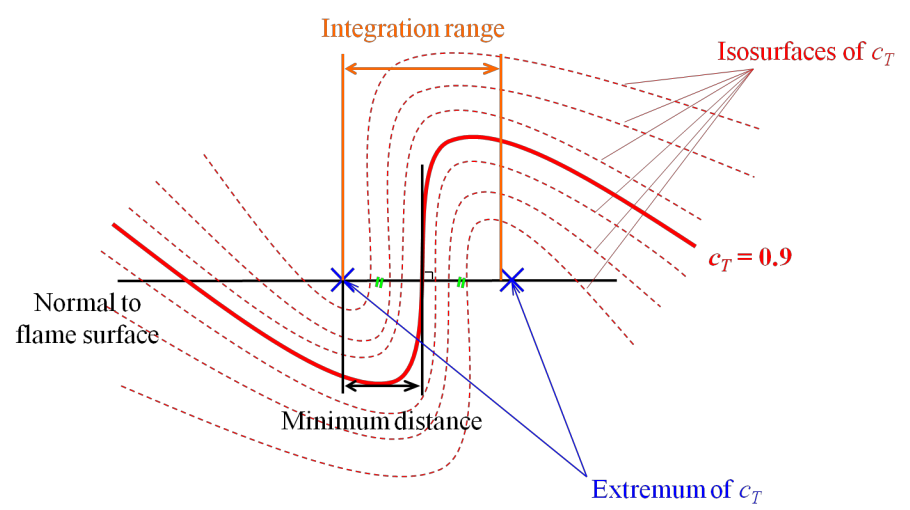

Figure 4. Schematic of the integration range, Range 3.
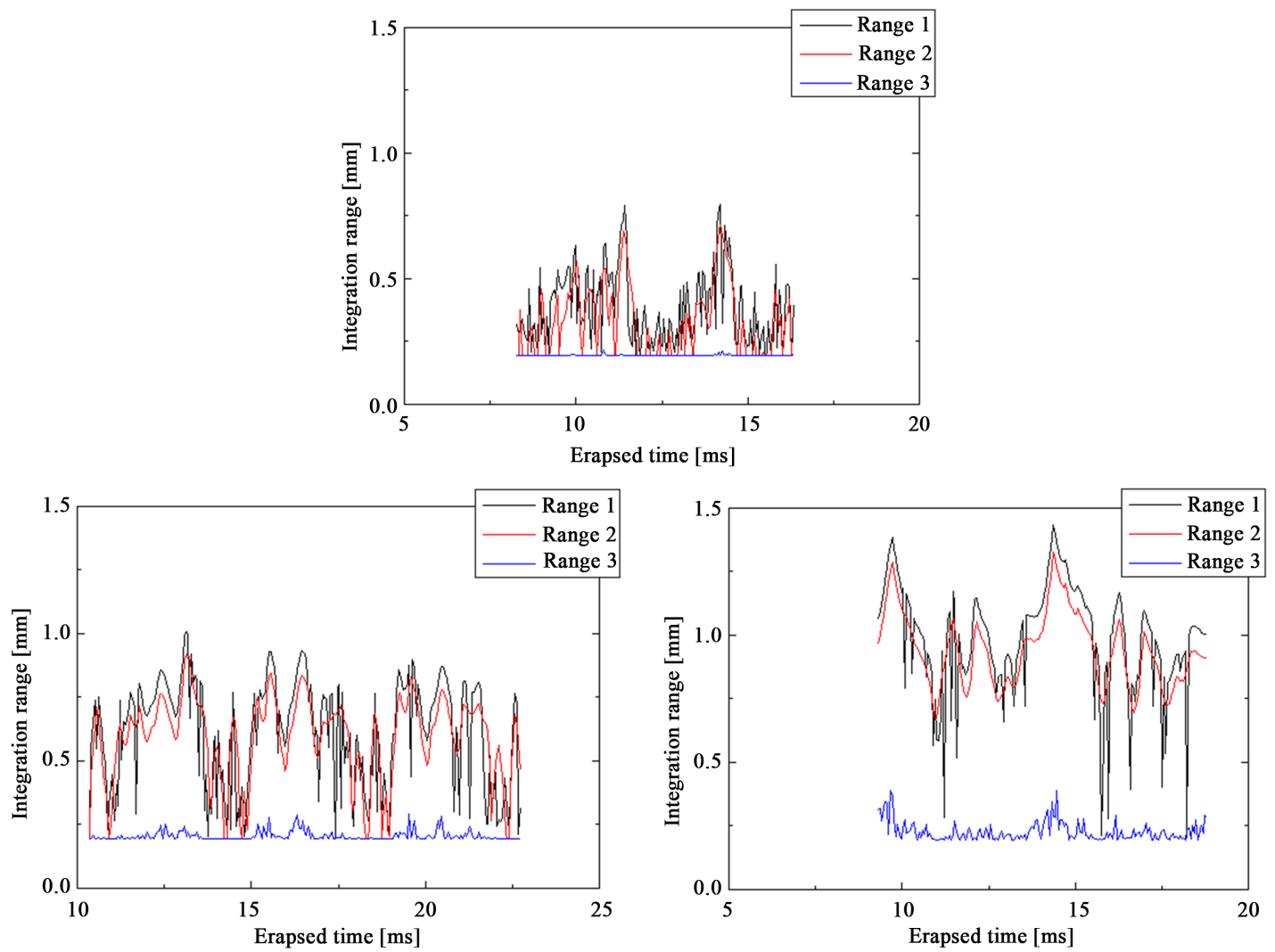

Figure 5. Temporal variations of the integration ranges of the local chemical reaction rates with the different Lewis numbers. From the top, case Ml, Mm, and Mh.

reaction rate on the point of the flame surface, thus the flame shape has the lower impact on Range 2 than Range 1. In contrast, the integration range in Range 3 was narrower and slightly fluctuated. Range 3 is defined directly by $c_{T}$, which is used for the definition of the flame surface, and thus Range 3 is little subject to the flame shape.

The probability density functions (pdfs) of $u_{L}$ evaluated by three kinds of the integration ranges with the different Lewis numbers are shown in Figure 6. $u_{L}$ was nondimensionalised by the unstretched laminar burning velocity, $u_{L}^{0}$. Note that very low peaks around 0.5 in case $\mathrm{Mm}$ and $\mathrm{Mh}$ are due to little inaccurate integration nearby the boundary of the computational domain. According to the other work [15], the reason why the profiles and modes of the pdfs were dependent on Lewis number was the deviation of the local chemical reaction rates along normal to the flame surface with the different Lewis numbers. In all Lewis number cases, the pdfs of Range 1 and Range 2 were almost the same profiles. In addition, the modes in Range 1 and Range 2 were lo- 

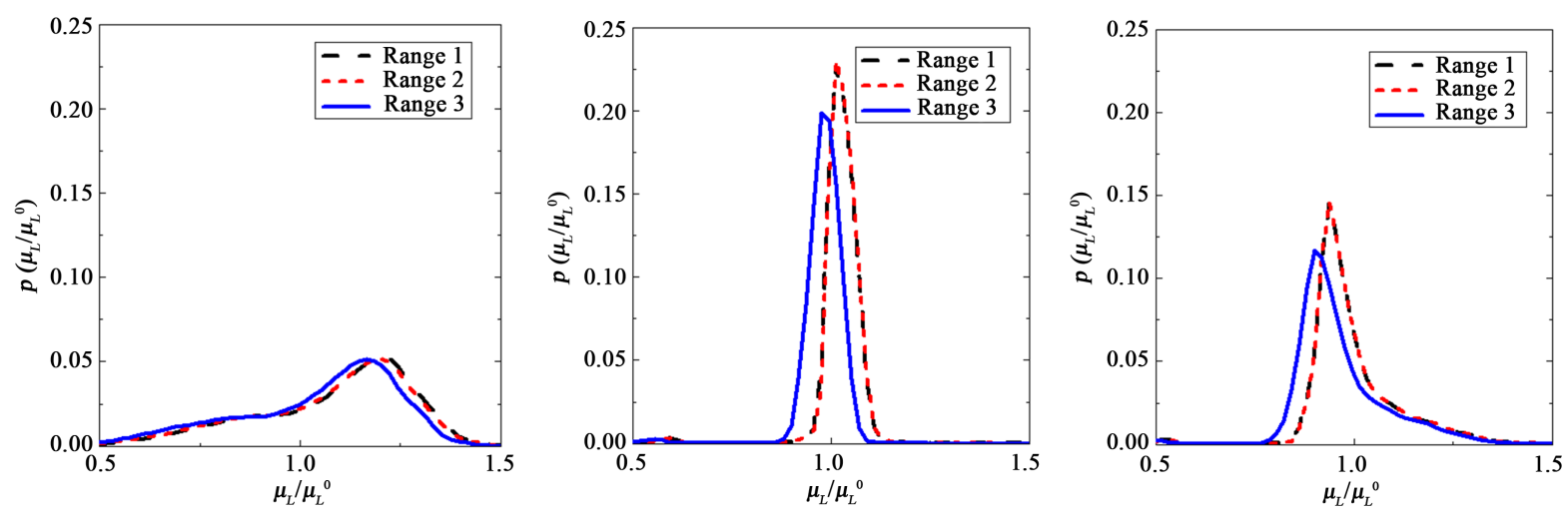

Figure 6. The probability density functions of $u_{L} / u_{L}^{0}$ evaluated by three kinds of the integration ranges with the different Lewis numbers. From the left, case $\mathrm{Ml}, \mathrm{Mm}$, and $\mathrm{Mh}$.

cated at larger $u_{L}$ than that in Range 3. The definitions of Range 1 and Range 2 are not subject to $c_{T}$, which is used for the definition of the flame surface. Thus, there are possibilities that Range 1 and Range 2 include the contributions of the chemical reactions at other points on the flame surface if the integration ranges in Range 1 and Range 2 pass close to other point on the flame surface. Therefore, the definitions of Range 1 and Range 2 lead to the overestimations of $u_{L}$. This is evident from the fact that the mode in Range 3 was located at $u_{L} / u_{L}^{0}$ $=1$ in the unity Lewis number case, where the thermal diffusion and the mass diffusion are balanced and thus the stability of the flame is neutral.

From the discussion above, the definition of Range 3 is considered to be appropriate to the evaluation of the local burning velocity in turbulent premixed flames under the conditions of this study.

\section{Conclusion}

The local burning velocity, which is considered to be the most appropriate definition of burning velocity in turbulent premixed flames, was evaluated by three kinds of the integration ranges using DNS data of turbulent premixed flames. It was found that the integration range defined by the directly prescribed reaction progress variable, which is used for the definition of the flame surface, is the most appropriate to the evaluation of the local burning velocity in turbulent premixed flames under the conditions of this study. The new technique to define the integration range, which was proposed in this study, is simple and accurate, and is considered to be available for the conditions of turbulent combustion that the flame thickness varies clearly.

\section{References}

[1] Poinsot, T., Echekki, T. and Mungal, M.G. (1992) A Study of the Laminar Flame Tip and Implications for Premixed Turbulent Combustion. Combustion Science and Technology, 81, 45-73. http://dx.doi.org/10.1080/00102209208951793

[2] Haworth, D.C. and Poinsot, T.J. (1992) Numerical Simulations of Lewis Number Effects in Turbulent Premixed Flames. Journal of Fluid Mechanics, 244, 405-436. http://dx.doi.org/10.1017/S0022112092003124

[3] Rutland, C.J. and Trouvé, A. (1993) Direct Simulations of Premixed Turbulent Flames with Nonunity Lewis Numbers. Combustion and Flame, 94, 41-57. http://dx.doi.org/10.1016/0010-2180(93)90018-X

[4] Chen, J.H. and Im, H.G. (2000) Stretch Effects on the Burning Velocity of Turbulent Premixed Hydrogen/Air Flames. Proceedings of the Combustion Institute, 28, 211-218. http://dx.doi.org/10.1016/S0082-0784(00)80213-1

[5] Bell, J.B., Cheng, R.K., Day, M.S. and Shepherd, I.G. (2007) Numerical Simulation of Lewis Number Effects on Lean Premixed Turbulent Flames. Proceedings of the Combustion Institute, 31, 1309-1317. http://dx.doi.org/10.1016/j.proci.2006.07.216

[6] Day, M., Tachibana, S., Bell, J., Lijewski, M., Beckner, V. and Cheng, R.K. (2012) A Combined Computational and Experimental Characterization of Lean Premixed Turbulent Low Swirl Laboratory Flames I. Methane Flames. Combustion and Flame, 159, 275-290. http://dx.doi.org/10.1016/j.combustflame.2011.06.016

[7] Han, I. and Huh, K.Y. (2008) Roles of Displacement Speed on Evolution of Flame Surface Density for Different Turbulent Intensities and Lewis Numbers in Turbulent Premixed Combustion. Combustion and Flame, 152, $194-205$. 
http://dx.doi.org/10.1016/j.combustflame.2007.10.003

[8] Tsuboi, K., Nishiki, S. and Hasegawa, T. (2008) An Analysis of Local Quantities of Turbulent Premixed Flames Using DNS Databases. Journal of Thermal Science and Technology, 3, 103-111. http://dx.doi.org/10.1299/jtst.3.103

[9] Poinsot, T.J. and Lele, S.K. (1992) Boundary Conditions for Direct Simulations of Compressible Viscous Flows. Journal of Computational Physics, 101, 104-129. http://dx.doi.org/10.1016/0021-9991(92)90046-2

[10] Baum, M., Poinsot, T. and Thévenin, D. (1995) Accurate Boundary Conditions for Multicomponent Reactive Flows. Journal of Computational Physics, 116, 247-261. http://dx.doi.org/10.1006/jcph.1995.1024

[11] Peters, N. (1999) The Turbulent Burning Velocity for Large-Scale and Small-Scale Turbulence. Journal of Fluid Mechanics, 384, 107-132. http://dx.doi.org/10.1017/S0022112098004212

[12] Nishiki, S., Hasegawa, T., Borghi, R. and Himeno, R. (2002) Analyzing and Modeling of Transport Properties of Turbulent Kinetic Energy and Turbulent Scalar Flux in Turbulent Premixed Flames by DNS. Journal of the Combustion Society of Japan, 48, 47-57. (in Japanese)

[13] Nishiki, S., Hasegawa, T., Borghi, R. and Himeno, R. (2006) Modelling of Turbulent Scalar Flux in Turbulent Premixed Flames Based on DNS Databases. Combustion Theory and Modelling, 10, 39-55. http://dx.doi.org/10.1080/13647830500307477

[14] Nishiki, S. (2003) DNS and Modeling of Turbulent Premixed Combustion. Doctoral Thesis, Nagoya Institute of Technology, Nagoya.

[15] Tsuboi, K., Tomita, E. and Hasegawa, T. (2014) DNS Analysis on the Correlation between Local Burning Velocity and Flame Displacement Speed of Turbulent Premixed Flames. Journal of Thermal Science and Technology (Submitted). 
Scientific Research Publishing (SCIRP) is one of the largest Open Access journal publishers. It is currently publishing more than 200 open access, online, peer-reviewed journals covering a wide range of academic disciplines. SCIRP serves the worldwide academic communities and contributes to the progress and application of science with its publication.

Other selected journals from SCIRP are listed as below. Submit your manuscript to us via either submit@scirp.org or Online Submission Portal.
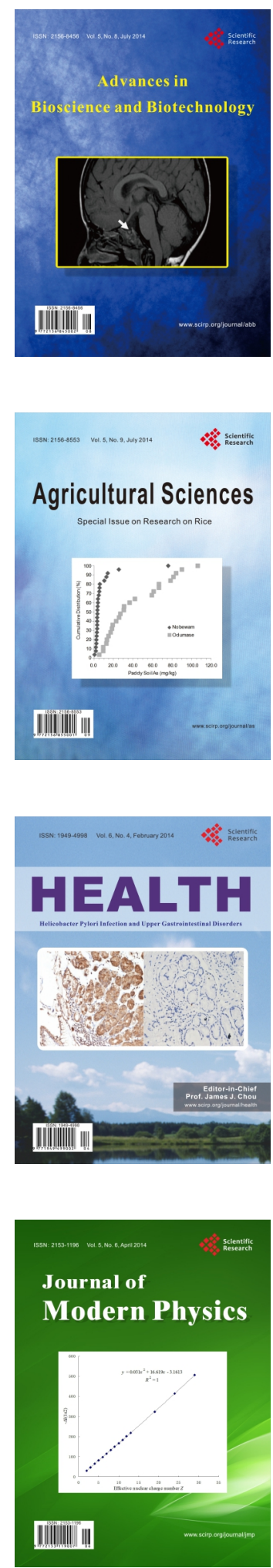
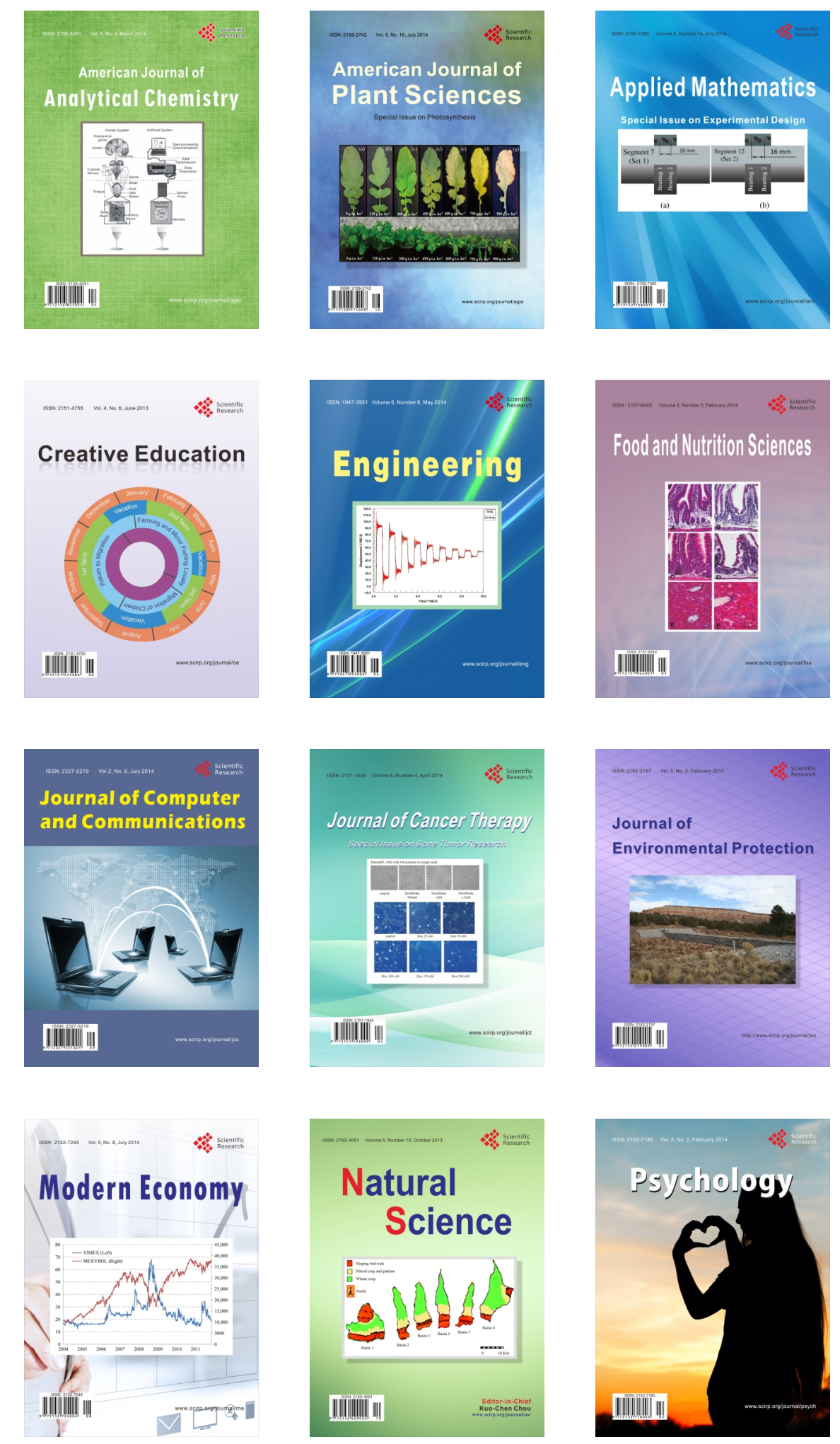